\title{
Free Convection MHD Couette Flow with Application of Periodic Temperature and Constant Heat Flux on Walls
}

\author{
Mustafa Rashied Abdullah \\ Faculty of Engineering \\ Al-Ahliyya Amman University \\ Amman, Jordan \\ mrashied@ammanu.edu.jo
}

\author{
Nidhal Saada \\ Faculty of Engineering \\ Al-Ahliyya Amman University \\ Amman, Jordan \\ n.saada@ammanu.edu.jo
}

\begin{abstract}
Numerical analysis and analytical solution were performed to study the free convection in transient Couette flow of an electrically conducting fluid confined between two vertical parallel plates. Constant heat flux on the wall with uniform vertical motion in its own plane and periodic temperature on the stationary wall were applied. The dimensionless governing momentum and energy equations are solved numerically using a fully implicit finite difference method. An analytical solution using eigenfunction expansion method is carried out for temperature profile in case of constant plate temperature. Analytical and numerical results converge at a satisfactory degree. The effect of different physical parameters on the transient velocity and temperature, such as Grashof's number (Gr), magnetic parameter (M), Prandtl number (Pr) and temperature frequency are also studied. It is found that the velocity increases with an increase in $\mathrm{Gr}$ and time, while it decreases with an increase in $\mathrm{Pr}$ and $M$.
\end{abstract}

Keywords-magnetohydrodynamics (MHD); Couette flow; free convection

\section{INTRODUCTION}

Magnetohydrodynamic (MHD) flow of a viscous incompressible electrically conducting fluid has many industrial and engineering applications. One of the main MHD fluid dynamics problems is the Couette flow, in which one of the two parallel plates is moving relatively to the other. Some important applications of the Couette flow are MHD power generators and pumps, aerodynamic heating, electrostatic precipitation, polymer technology and petroleum industry. The effect of the magnetic field on the natural convection flow between two vertical plates under various conditions has been studied extensively. For instance, the Laplace transform technique was used to obtain expressions for velocity and temperature fields and their related quantities for the unsteady free convective flow of an incompressible viscous fluid between two vertical parallel plates for impulsive start of one of the plates [1]. The combined effect of natural convection and uniform transverse magnetic field on the Couette flow of an electronically conducting fluid between two parallel plates for impulsive motion of one of the plates was studied in [2], in which expressions for velocity and temperature fields were presented for two different cases, under the assumption of negligible induced magnetic field. The effects of magnetic interaction number, slip factor and relative temperature difference on velocity and temperature profiles, and entropy generation in MHD flow of a fluid with variable properties over a rotating disk was studied in [3], where velocities, temperature profiles, and average entropy generation rate were obtained for different parameter values [3]. The effect of the magnetic field and of the internal heat generation on the free convection flow in a rectangular cavity filled with a porous medium was investigated in [4]. Results indicated that there is an inverse relationship between the Nusselt number and the Hartmann number. The reverse occurs with an increase in magnetic field inclination angle. The impact of a varying magnetic field on forced convective heat transfer was investigated in [5]. Results indicated an increase in the Kelvin forces associated with a thermal boundary layer thickness decrease (higher Reynolds Number).

The Couette flow of an electrically conducting fluid between two parallel plates in the presence of a transverse magnetic field in case of constant heat flux has been studied extensively. A closed form solution for the transient free convection flow of a viscous fluid between two infinite vertical parallel plates in the presence of radiation was presented in [6]. The flow is set up due to the free convective currents occurring as a result of the application of constant heat flux at one wall and constant temperature on the other wall. An exact solution to the problem of natural convection in unsteady Couette flow between two long vertical parallel plates in the presence of constant heat flux and thermal radiation was presented in [7]. It was found that the configuration of the flow field is significantly effected significantly by the interaction between radiation, buoyancy forces and the applied shear force induced by a uniform vertical motion of the hot wall. Authors in [8] studied the unsteady oscillatory Couette flow between vertical parallel plates with constant radiative heat flux for an optically thick fluid. The perturbation method was used to find an analytical solution for the velocity and temperature functions and it was found that the increasing of the thermal radiation parameter increases the fluid flow of thick fluids such as blood.

Corresponding author: Mustafa Rashied Abdullah 
Other studies were carried out in order to investigate the effect of varying plate temperature on the behavior of Couette flow [9-13]. The effect of free convection on heat transfer and flow through a highly porous medium bounded by two vertical parallel porous plates assuming periodic temperature at the moving plate was investigated in [9]. Authors in [10] investigated the problem of MHD free convection and oscillatory flow of an optically thin fluid bounded by two horizontal porous parallel walls under the influence of an external imposed transverse magnetic field in a porous medium with periodic wall temperature. An exact solution to the problem of unsteady free convection flow and heat transfer in a viscous incompressible fluid between two long vertical parallel plates was presented in [11]. The temperature at one of the plates increases linearly with time while that at the other plate remains constant at the initial fluid temperature. Authors in [12] studied the effect of free convection on the heat transfer and the flow through a highly porous medium bounded by two vertical parallel porous plates in presence of a transverse magnetic field. The approximate solutions for velocity and temperature were obtained by assuming periodic temperature at the moving plate. Authors in [13] studied the magnetic field effect on an unsteady free convection flow of a viscous incompressible electrically conducting fluid with dissipative heat between two long vertical parallel plates where the temperature of one of the plates oscillates about a constant nonzero mean temperature. Radiation effects on free convection MHD Couette flow with variable wall temperature in the presence of heat generation have been studied in [14]. Authors in [15] analyzed the effects of thermal radiation and constant mass diffusion on the transient laminar free convective flow in a vertical channel with variable temperature. Authors in [16] studied the MHD effect on unsteady free convection between a pair of infinite vertical Couette plates. The temperature of the plates was assumed to vary with time.

The objective of this study is to investigate the transient free convection MHD Couette flow with the presence of both periodic temperature and constant heat flux on walls. A numerical analysis of the dimensionless equations is performed and verified by an analytical solution in case of constant plate temperature.

\section{MATHEMATICAL FORMULATION}

Consider a two-dimensional MHD flow of an incompressible electrically conducting viscous fluid between two long vertical parallel plates separated by a distance $h$ as shown in Figure 1. A conductive liquid with density $\rho$, dynamic viscosity $\mu$, and electrical conductivity $\sigma$ fills the region between the plates. The coordinates $x, y$ are aligned, respectively, along the plate axis, and width. The plate is placed in a uniform transverse magnetic field of flux density $B_{o}$ in the $y$ direction. At time $t \leq 0$, the two walls and the fluid are assumed to have the same stationary temperature $T_{0}$. At time $t>0$, the plate at $y=0$ starts moving with a constant velocity and a constant heat flux, while the plate at $y=h$ is heated with periodic temperature and maintained stationary. To setup the mathematical model of the problem, the following assumptions are made:

- The fluid physical properties are constant.
- The fluid flow is unsteady, laminar, incompressible, viscous, electrically conducting and fully developed.

- The magnetic Reynolds numbers are small, hence the induced magnetic field is negligible.

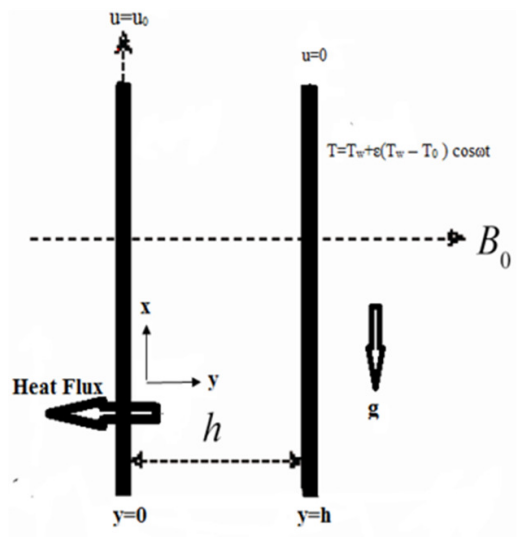

Fig. 1. Schematic diagram of the system

Based on the above assumptions and applying the magnetic field with a presence of periodic plate temperature, the transient governing equations of the MHD flow that present the fluid motion and temperature are:

$$
\begin{aligned}
& \frac{\partial u}{\partial t}=g \beta\left(T-T_{0}\right)+v \frac{\partial^{2} u}{\partial y^{2}}-\frac{\sigma B_{0}^{2}}{\rho} u \\
& \rho c_{p} \frac{\partial T}{\partial t}=k \frac{\partial^{2} T}{\partial y^{2}}
\end{aligned}
$$

where $u$ is the axial velocity, $T$ is the fluid temperature, $v$ is the kinematic viscosity, $k$ is the thermal conductivity, and $C_{p}$ is the speicific heat, with initial and boundary conditions:

$$
\begin{aligned}
& t \leq 0: u=0 \quad T=T_{0} \text { for all y } t>0: \\
& u=u_{0} \quad \frac{\partial T}{\partial y}=-\frac{q}{k} \quad \text { at } y=0 \\
& u=0 \quad T=T_{w}+\epsilon\left(T_{w}-T_{0}\right) \cos \bar{\omega} t \quad \text { at } y=h
\end{aligned}
$$

To write the governing equations in dimensionless form, the following dimensionless variables are introduced:

$$
\begin{aligned}
& Y=\frac{y}{h}, \quad \tau=\frac{v t}{h^{2}}, \quad \omega=\frac{h^{2} \bar{\omega}}{v}, U=\frac{u}{u_{0}}, \theta=\frac{T-T_{0}}{T_{w}-T_{0}}, \\
& M=\frac{\sigma h^{2} B_{0}^{2}}{\mu}, G r=\frac{g \beta h^{2}\left(T_{w}-T_{0}\right)}{v u_{0}}, \quad \operatorname{Pr}=\frac{\mu c_{p}}{k}
\end{aligned}
$$

The dimensionless equations become:

$$
\begin{aligned}
& \frac{\partial U}{\partial \tau}=G r \theta+\frac{\partial^{2} U}{\partial Y^{2}}-M U \\
& \frac{\partial \theta}{\partial \tau}=\frac{1}{\operatorname{Pr}} \frac{\partial^{2} \theta}{\partial Y^{2}}
\end{aligned}
$$

The corresponding boundary conditions can be specified as:

$$
\begin{array}{ll}
\tau \leq 0: \quad U=0, \quad \theta=0 \text { for all } \mathrm{Y} \\
\tau>0: \quad U=1, \quad \frac{\partial \theta}{\partial Y}=-1 \quad \text { at } Y=0 \\
& U=0, \quad \theta=1+\varepsilon \cos (\omega \tau) \quad \text { at } Y=1
\end{array}
$$




\section{NUMERICAL ANALYSIS}

Finite difference technique is applied to solve the dimensionless momentum and energy equations to determine the velocity and temperature distributions for different parameter values. A uniform grid consisting of a large number of nodes in the $y$ direction is used. Crank-Nicolson method is used to solve the equations with a large amount of time steps. Crank-Nicolson method is fully implicit and numerically stable. It has a higher order of accuracy when the solutions are directly obtained from the Thomas algorithm.

\section{ANALYTICAL SOLUTION}

The numerical solution can be verified by its comparison with the analytical solution for the case of constant wall temperature $(\varepsilon=0)$. The analytical eigenfunction expansion method is used to solve the energy equation. The dimensionless time dependent energy equation can be written as:

$$
\frac{\partial \theta}{\partial \tau}=\frac{1}{\operatorname{Pr}} \frac{\partial^{2} \theta}{\partial Y^{2}}
$$

The boundary conditions for constant wall temperature can be written as follows:

$$
\begin{array}{cl}
\tau \leq 0: & \theta=0 \text { for all } \mathrm{Y} \\
\tau>0: & \frac{\partial \theta}{\partial Y}=-1 \quad \text { at } Y=0 \\
& \theta=1 \text { at } Y=1
\end{array}
$$

Since the boundary conditions are non homogeneous, we can convert them to homogeneous by introducing:

$$
F(Y, \tau)=\theta(Y, \tau)-(2-Y)
$$

Then the energy equation becomes:

$$
\frac{\partial F}{\partial \tau}=\frac{1}{P r} \frac{\partial^{2} F}{\partial Y^{2}}
$$

And the boundary conditions can be specified as:

$$
\begin{array}{cl}
\tau \leq 0: & F=(Y-2) \quad \text { for all } \mathrm{Y} \\
\tau>0: & \frac{\partial F}{\partial Y}=0 \quad \text { at } Y=0 \\
& \mathrm{~F}=0, \quad \text { at } Y=1
\end{array}
$$

Let $F(Y, \tau)=\varnothing(Y) \delta(\tau)$. Taking the derivatives and substituting into (11) yields the eigenvalue problem as:

$$
\frac{d^{2} \emptyset}{d Y^{2}}+\lambda \emptyset=0 \quad \frac{\partial \phi}{\partial Y}(0)=\emptyset(1)=0
$$

where $\lambda$ is the separation constant. The solution of the above equation will be:

$$
\emptyset_{n}(Y)=\cos (\sqrt{\lambda} Y)
$$

with eigenvalues:

$$
\lambda_{n}=\left(\frac{2 n-1}{2} \pi\right)^{2}
$$

For each $\mathrm{n}$, the solution for $\delta(\tau)$ is $\delta_{n}(\tau)=e^{-\frac{\lambda_{n} \tau}{p r}}$. Hence the series solution for $F(Y, \tau)$ is:

$$
F(Y, \tau)=\sum_{n=1}^{\infty} A_{n} \cos (\sqrt{\lambda} Y) e^{\frac{-\lambda}{p r} \tau}
$$

which satisfies the non-homogeneous initial condition $F(Y, 0)=(Y-2)$. Hence $A_{n}=\frac{-2}{\lambda}(1+\sqrt{\lambda} \sin (\sqrt{\lambda}))$, where $n=1,2, \ldots \ldots, \infty$. Then the final form of solution is:

$$
\begin{aligned}
& \theta(Y, \tau)=\left(\sum_{n=1}^{\infty} \frac{-2}{\lambda}(1+\sqrt{\lambda} \sin (\sqrt{\lambda})) \cos (\sqrt{\lambda} Y) e^{\frac{-\lambda}{p r} \tau}\right) \\
& +(2-Y)
\end{aligned}
$$
by:

The coefficient of heat transfer (Nusselt number), is given

$$
N u=\frac{h\left(\frac{\partial y}{\partial x}\right)}{\left(T-T_{0}\right)}
$$

Using the dimensionless temperature expression, the Nusselt number at moving and stationary plates can be written as:

$$
\begin{aligned}
& N u_{0}=\frac{-1}{\theta(0, \tau)}\left(\frac{\partial \theta}{\partial Y}\right)_{Y=0}=\frac{1}{\theta(0, \tau)} \\
& N u_{1}=-\left(\frac{\partial \theta}{\partial Y}\right)_{Y=1} \\
& =\left(\sum_{n=1}^{\infty}\left(\frac{2}{\sqrt{\lambda}} \sin (\sqrt{\lambda})+2 \sin ^{2}(\sqrt{\lambda})\right) e^{\frac{-\lambda}{p r}}\right)-1
\end{aligned}
$$

\section{RESULTS AND DISCUSSION}

In this section, the effect of different dimensionless parameters on the velocity and temperature profiles is discussed. The numerical solution using Crank-Niclson technique for velocity and temperature profiles is computed for different values of magnetic parameter, Prandtl number, Grashof number, and temperature frequency. The following parameter values are used to get the results: $G r=5, \operatorname{Pr}=7, M=1$, $\omega=10, \varepsilon=0.2, \tau=1$. Figure 2 illustrates the effect of Grashof number on the dimensionless velocity profile. It is seen that the effect of increasing $G r$ would be to increase the velocity $\mathrm{U}$ when all other parameters are held constant. Figure 3 shows the effect of magnetic field on the velocity profile which clearly indicates that the increase in the applied magnetic intensity would result in a decrease in velocity.

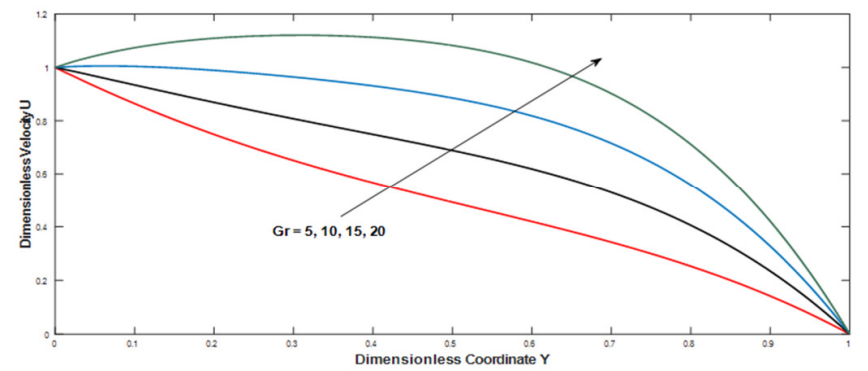

Fig. 2. Velocity profile for different Grashof numbers (Gr)

The effect of the Prandtl number Pr on the velocity profile is shown in Figure 4. It shows that increasing Pr would decrease the velocity. The effect of $\operatorname{Pr}$ on the temperature field is illustrated in Figure 5. It is observed that as Pr increases, the temperature in the fluid decreases. Also, it is seen that Pr has a 
significant influence on the temperature of the plate with constant heat flux for higher values of $\mathrm{Pr}$ as shown in Figure 5.

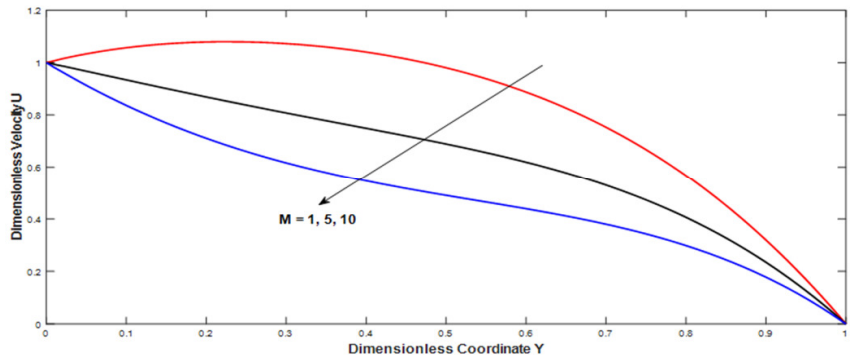

Fig. 3. Velocity profile for different magnetic parameter (M)

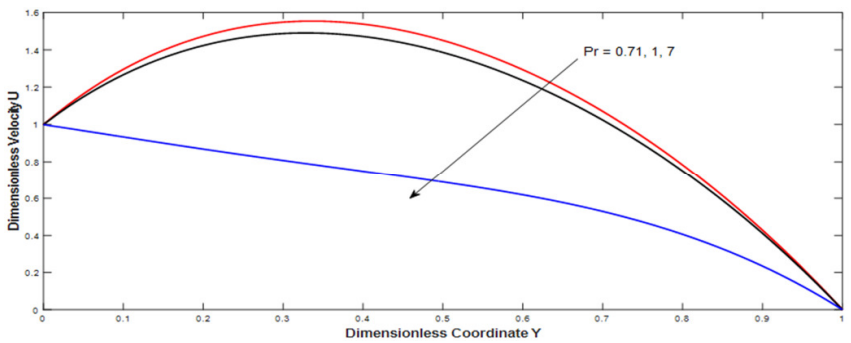

Fig. 4. Velocity profile for different Prandtl numbers (Pr)

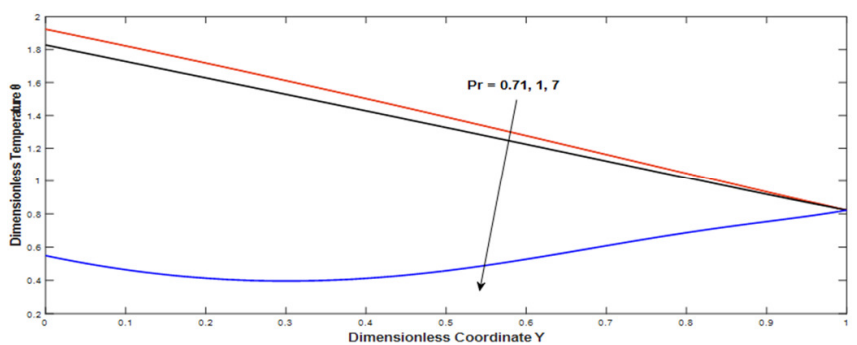

Fig. 5. Temperature profile for different Prandtl number (Pr)

The transient velocity and temperature profiles are shown in Figures 6 and 7 for different locations on the fluid. It is noticed that the effect of increasing time is to increase the velocity and temperature until they reach a steady state, and the required time is reduced as we move towards the stasionary plate which has a periodic temperature. It is noticed also that both velocity and temperature have oscillatory behavior with higher amplitude nearby the stationary plate which decays as we move away from the plate.

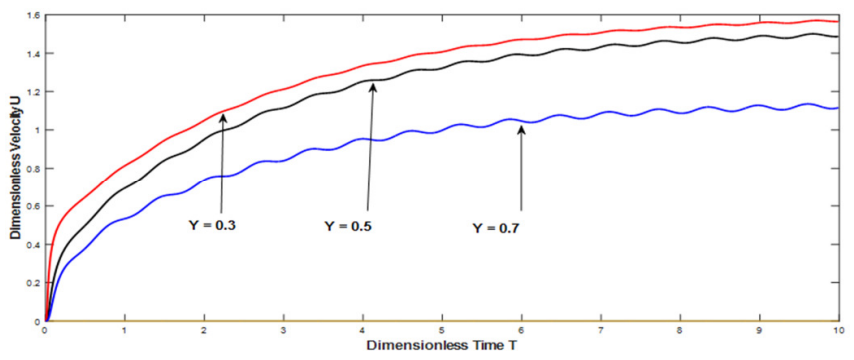

Fig. 6. Transient velocity at different points on the Y coordinate

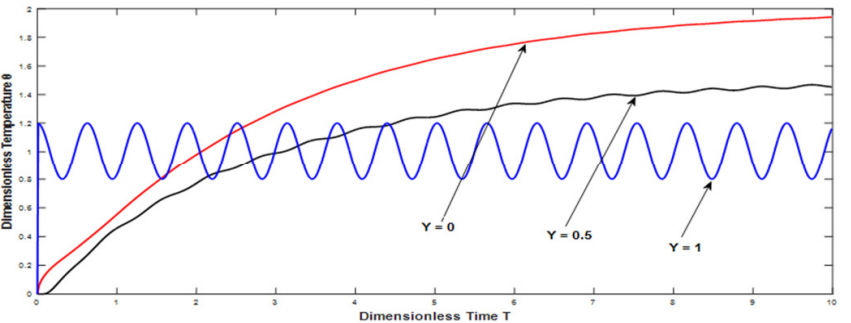

Fig. 7. Transient temperature at different points on the $\mathrm{Y}$ coordinate

To study the effect of plate temperature frequency on the transient temperature profile, the pulsed volume must be cleared. As shown in Figure 8, as the frequency increases, the temperature difference appears to fade away with time. Hence, it seems that the temperature at high frequency is continuous rather than oscilating.

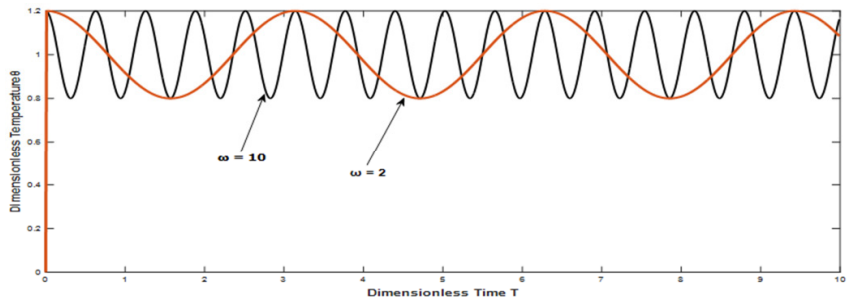

Fig. 8. Transient temperature for different values of temparature frequency

A comparison of the transient temperature results of the Crank-Niclson solution (fully implicit method) with analytical solution in the case of $\varepsilon=0$ for temperature profile is shown in Figure 9. It is seen that the results are in good agreement with each other.

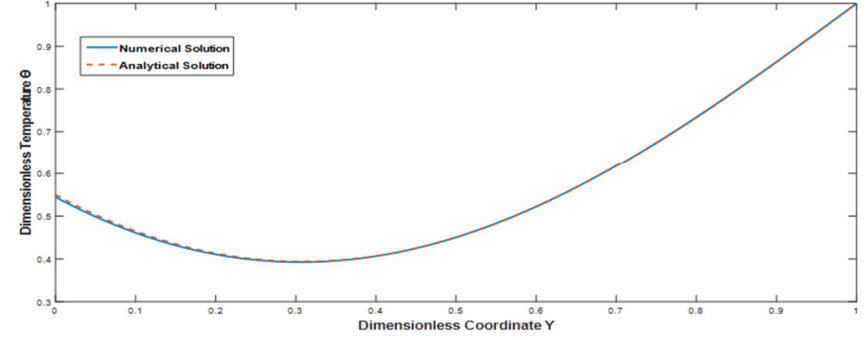

Fig. 9. Comparison of temperature numerical and analytical results

\section{CONCLUSIONS}

A fully implicit numerical solution to the problem of transient free convective Couette flow of a viscous incompressible fluid confined between two vertical parallel plates in the presence of constant heat flux and periodic temperature on the walls has been prersented. The dimensionless governing partial differential equations are solved by the Crank-Niclson technique and verified by an eigen function expansion method. The effect of different parameters such as magnetic parameter, Grashof number, Prandtl number and time are studied. The conclusions of the study are: 
- The effect of MHD on the fluid flow appears through the magnetic parameter effect. Magnetic field exerts a retarding influence on the fluid velocity, which means that a slightly conductive fluid is not affected by the presence of the magnetic field. That could mean that control of the flow can be obtained by controlling the magnetic flux and by a good choice of electrical conductivity.

- Increasing the Grashof number will cause an increase on velocity.

- Increasing the Prandtl number will decrease velocity and temperature.

- The periodic behavior of the plate temperature is reflected on transient velocity and temperature profiles.

\begin{tabular}{|c|c|}
\hline & NOMENCLATURE \\
\hline Bo & magnetic flux density $(\mathrm{T})$ \\
\hline$C p$ & specific heat, $\left(\mathrm{J} \mathrm{kg}^{-1} \cdot \mathrm{K}^{-1}\right)$ \\
\hline$G r$ & Grashof number \\
\hline$K$ & thermal conductivity, $\left(\mathrm{W} \cdot \mathrm{m}^{-1} \cdot \mathrm{K}^{-1}\right)$ \\
\hline$M$ & magnetic parameter \\
\hline $\operatorname{Pr}$ & Prandtl number \\
\hline$T$ & time, $(\mathrm{s})$ \\
\hline$T$ & temperature, $(\mathrm{K})$ \\
\hline$T_{w}$ & wall temperature, $(\mathrm{K})$ \\
\hline$T_{0}$ & initial fluid temperature, $(\mathrm{K})$ \\
\hline$u$ & velocity component in the $\mathrm{x}$ direction, $\left(\mathrm{m} \cdot \mathrm{s}^{-1}\right)$ \\
\hline$U$ & dimensionless velocity \\
\hline$x, y$ & cartesian coordinates \\
\hline$Y$ & dimensionless coordinate \\
\hline$\varepsilon$ & small reference parameter \\
\hline $\bar{\omega}$ & frequency of oscillation, $\left(\right.$ rad. $\left.\mathrm{s}^{-1}\right)$ \\
\hline$\omega$ & dimensionless frequency \\
\hline$\varphi, \delta$ & separation variables \\
\hline$\theta$ & dimensionless temperature \\
\hline$\tau$ & dimensionless time \\
\hline$\lambda$ & separation constant \\
\hline$v$ & kinematic viscosity, $\left(\mathrm{m}^{2} \cdot \mathrm{s}^{-1}\right)$ \\
\hline$\sigma$ & electrical conductivity, (siemens. $\mathrm{m}^{-1}$ ) \\
\hline$\rho$ & density, $\left(\mathrm{kg} \cdot \mathrm{m}^{-3}\right)$ \\
\hline$\mu$ & dynamic viscosity, $\left(\mathrm{kg} \cdot \mathrm{m}^{-1} \cdot \mathrm{s}^{-1}\right)$ \\
\hline
\end{tabular}

\section{REFERENCES}

[1] A. Singh, "Natural convection in unsteady Couette motion", Defence Science Journal, Vol. 38, No. 1, pp. 35-41, 1988

[2] B. Jha, "Natural convection in unsteady MHD Couette flow", Heat and Mass Transfer, Vol. 37, No. 4, pp. 329-331, 2001

[3] M. M. Rashidi, N. Kavyani, S. Abelman, "Investigation of entropy generation in MHD and slip over a rotating porous disk with variable properties", International Journal of Heat and Mass Transfer, Vol. 70, pp. 892-917, 2014

[4] A. M. Rashad, M. M. Rashidi, G. Lorenzini, S. E. Ahmed, A. M. Aly, "Magnetic field and internal heat generation effects on the free convection in a rectangular cavity filled with a porous medium saturated with $\mathrm{Cu}-$ water nanofluid", International Journal of Heat and Mass Transfer, Vol. 104, pp. 878-89, 2017

[5] M. Sheikholeslami, K. Vajravelu, M. M. Rashidi, "Forced convection heat transfer in a semi annulus under the influence of a variable magnetic field", International Journal of Heat and Mass Transfer, Vol. 92, pp. 339-348, 2016

[6] R. Chaudhary, P. Jain, "Exact solutions of incompressible couette flow with constant temperature and constant heat flux on walls in the presence of radiation", Turkish Journal of Engineering and Environmental Sciences, Vol. 31, No. 5, pp. 297-304, 2007

[7] M. Narahari, "Effects of thermal radiation and free convection currents on the unsteady couette flow between two vertical parallel plates with constant heat flux at one boundary", Wseas Transactions on Heat and Mass Transfer, Vol. 5, No. 1, pp. 21-30, 2010
[8] K. Bunonyo, E. Amos, I. C. Eli, "Unsteady oscillatory couette flow between vertical parallel plates with constant radiative heat flux", Asian Research Journal of Mathematics, Vol. 11, No. 2, pp. 1-11, 2018

[9] P. Sharma, B. Sharma, R. C. Tamkang, "Unsteady free covection oscillatory couette flow through a porous medium with periodic wall temperature", Journal of Mathematics, Vol. 38, No. 1, pp. 93-102, 2007

[10] C. Israel-Cookey, E. Amos, C. Nwaigwe, "MHD oscillatory couette flow of a radiating viscous fluid in a porous medium with periodic wall temperature", American Journal of Scientific and Industrial Research, Vol. 1, No. 2, pp. 326-331, 2010

[11] M. Narahari, B. Dutta, "Free convection flow and heat transfer between two vertical parallel plates with variable temperature at one boundary", Acta Technica, Vol. 56, pp. 103-113, 2011

[12] M. Raju, S. Varma, "Unsteady mhd free convection oscillatory couette flow through a porous medium with periodic wall temperature", IManager's Journal on Future Engineering \& Technology, Vol. 6, No. 4, pp. 7-12, 2011

[13] N. Ahmed, K. Sarma, D. P. Barua, "Magnetic field effect on free convective oscillatory flow between two vertical parallel plates with periodic plate temperature and dissipative heat", Applied Mathematical Sciences, Vol. 6, No. 39, pp. 1913-1924, 2012

[14] S. Das, B. Sarkar, R. N. Jana, "Radiation effects on free convection mhd couette flow started exponentially with variable wall temperature in presence of heat generation", Open Journal of Fluid Dynamics, Vol. 2, No. 1, pp. 14-27, 2012

[15] H. K. Mandal, S. Das, R. N. Jana, "Transient free convection in a vertical channel with variable temperature and mass diffusion", Chemical and Process Engineering Research, Vol. 23, pp. 38-54, 2014

[16] B. Zigta, P. Koya, "The effect of mhd on free convection with periodic temperature and concentration in the presence of thermal radiation and chemical reaction", International Journal of Applied Mechanics and Engineering, Vol. 22, No.4, pp. 1059-1073, 2017 